\title{
Lobular Capillary Hemangioma of the Nasal Cavity: A Retrospective Study of 15 Cases in Taiwan
}

\author{
Tzu-Hang Chi ${ }^{1,2}$, Chien-Han Yuan², Shang-Tao Chien ${ }^{3}$ \\ ${ }^{1}$ Department of Otolaryngology, Taoyuan Armed Forces General Hospital, Taoyuan, Taiwan \\ ${ }^{2}$ Department of Otolaryngology, Kaohsiung Armed Forces General Hospital, Kaohsiung, Taiwan \\ ${ }^{3}$ Department of Pathology, Kaohsiung Armed Forces General Hospital, Kaohsiung, Taiwan
}

Background: Lobular capillary hemangioma of the nasal cavity is an uncommon benign vascular tumor of unknown etiology. There have been only very few case reports in Taiwan.

Aims: This study aimed to analyze the clinical features, radiological findings, treatment modalities, and outcome of lobular capillary hemangioma treated at a teaching hospital in Taiwan during a period of 10 years.

Study Design: Descriptive study.

Methods: Retrospective chart reviews were performed on patients who were diagnosed with lobular capillary hemangioma of the nasal cavity at Kaohsiung Armed Forces General Hospital, Kaohsiung, Taiwan, from January 2003 to December 2012. Data retrieved included age, gender, clinical symptoms, computed tomography (CT) findings, treatment modalities, and outcome for further analysis.

Results: Of the 15 patients identified, there were five males and ten females ranging from 17 to 86 years of age, with a mean age of
43.8 \pm 20.2 . Epistaxis was the most common presenting symptom. All patients presented a unilateral nasal lobular capillary hemangioma. The most commonly affected site was the anterior nasal septum, followed by the inferior turbinate, vestibule, middle turbinate, and posterior nasal septum. All lesions presented as soft tissue density without bony erosions under CT examination. Endoscopic excisional surgery $(n=12)$ or classical local excision $(n=3)$ was performed for complete removal of the hemangioma. No evidence of recurrence was observed with 6 to 75 months of follow-up.

Conclusion: Lobular capillary hemangioma of the nasal cavity was usually found to occur in anterior septum with epistaxis. Complete excision with endoscopic surgery or classical local excision was recommended and recurrence can be prevented.

(Balkan Med J 2014;31:69-71).

Key Words: Endoscopic surgery, epistaxis, lobular capillary hemangioma, nasal cavity
Hemangiomas are benign tumors made up of blood vessels that most commonly occur in the skin, mucosa, and internal organs such as the brain and liver. Hemangiomas are categorized to capillary, cavernous and mixed types according to the histopathological features. Lobular capillary hemangioma, also known as pyogenic granuloma, is uncommonly seen localized in the nasal cavity (1). Current information on this disease is mostly derived from case series of western populations, and little is known about this disease in Asians. In the present study, 15 patients diagnosed and treated with lobular capillary hemangioma of the nasal cavity at a teaching hospital in Taiwan were reviewed. The clinical features, radiological findings, treatment modalities, and outcomes were analyzed.

\section{MATERIAL AND METHODS}

A retrospective chart review of patients who were diagnosed with lobular capillary hemangioma of the nasal cavity between January
2003 and December 2012 at a teaching hospital in Taiwan was performed. Patients who had undergone biopsy procedures without surgical excision were excluded from this study. Information on patient age, gender, clinical symptoms, CT findings, treatment modalities, and outcomes was collected for analysis.

\section{RESULTS}

There were 15 patients enrolled in this study, including five males $(33.3 \%)$ and ten females $(66.7 \%)$ with a male:female ratio of 1:2. The mean age was $43.8 \pm 20.2$, ranging from 17 to 86 (Table 1). Approximately $73.3 \%$ (11/15) of the patients were between 20 and 60 years of age. Twelve patients had no underlying diseases, whereas the remaining three had diabetes mellitus, hypertension, and rheumatoid arthritis.

All patients presented with unilateral lobular capillary hemangioma of the nasal cavity with $60.0 \%(9 / 15)$ of the lesions on the left side and $40.0 \%(6 / 15)$ on the right. The 
TABLE 1. Demographic characteristics of patients with lobular capillary hemangiomas of the nasal cavity

\begin{tabular}{|c|c|c|c|c|c|c|c|}
\hline No. & Gender & $\begin{array}{l}\text { Age } \\
\text { (year) }\end{array}$ & $\begin{array}{l}\text { Duration } \\
\text { of symptoms }\end{array}$ & Symptoms & Lesion site & Treatment\# & $\begin{array}{l}\text { Follow-up } \\
\text { (month) }\end{array}$ \\
\hline 1 & Female & 45 & 3 weeks & Nasal obstruction, rhinorrhea, protruding tumor & $\mathrm{R}, \mathrm{V}$ & CLE & 8 \\
\hline 2 & Male & 30 & 2 weeks & Epistaxis, nasal obstruction, & $\mathrm{L}, \mathrm{AS}$ & EES & 6 \\
\hline 3 & Female & 31 & 2 weeks & Epistaxis, rhinorrhea & L, IT & EES & 12 \\
\hline 4 & Female & 65 & 5 months & Epistaxis, nasal obstruction, & L, PS & EES & 24 \\
\hline 5 & Female & 17 & 3 months & Epistaxis, rhinorrhea & L, IT & EES & 43 \\
\hline 6 & Female & 58 & 1 month & Epistaxis, nasal obstruction & $\mathrm{L}, \mathrm{AS}$ & EES & 28 \\
\hline 7 & Female & 86 & 1 year & Epistaxis, nasal obstruction, facial pain & $\mathrm{R}, \mathrm{IT}$ & EES & 47 \\
\hline 8 & Male & 23 & 2 weeks & Epistaxis, nasal obstruction, protruding tumor & $\mathrm{L}, \mathrm{V}$ & CLE & 42 \\
\hline 9 & Male & 22 & 3 weeks & Epistaxis, nasal obstruction, protruding tumor & $\mathrm{R}, \mathrm{V}$ & CLE & 41 \\
\hline 10 & Female & 75 & 1 year & Epistaxis, nasal obstruction, rhinorrhea & $\mathrm{R}, \mathrm{AS}$ & EES & 75 \\
\hline 11 & Male & 22 & 2 months & Epistaxis, nasal obstruction, rhinorrhea & L, IT & EES & 7 \\
\hline 12 & Female & 54 & 3 weeks & Epistaxis, nasal obstruction, rhinorrhea & $\mathrm{L}, \mathrm{AS}$ & EES & 24 \\
\hline 13 & Female & 52 & 8 months & Epistaxis, nasal obstruction, rhinorrhea & $\mathrm{L}, \mathrm{AS}$ & EES & 15 \\
\hline 14 & Female & 41 & 1 month & Epistaxis, rhinorrhea, facial pain, headache & $\mathrm{R}, \mathrm{MT}$ & EES & 27 \\
\hline 15 & Male & 36 & 3 months & Epistaxis, nasal obstruction & $\mathrm{R}, \mathrm{AS}$ & EES & 11 \\
\hline
\end{tabular}

R: right; L:left; V: vestibule; AS: anterior septum; PS: posterior septum; MT: middle turbinate; IT: inferior turbinate; CLE: classical local excision; EES: endoscopic excisional surgery

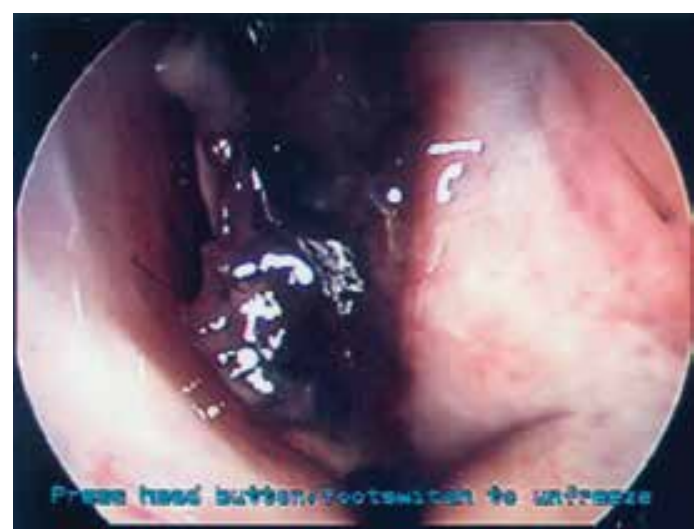

FIG. 1. Rigid endoscopy of the patient showing one dark-red polypoid mass with a blood clot arising from the posterior nasal septum of the left nasal cavity

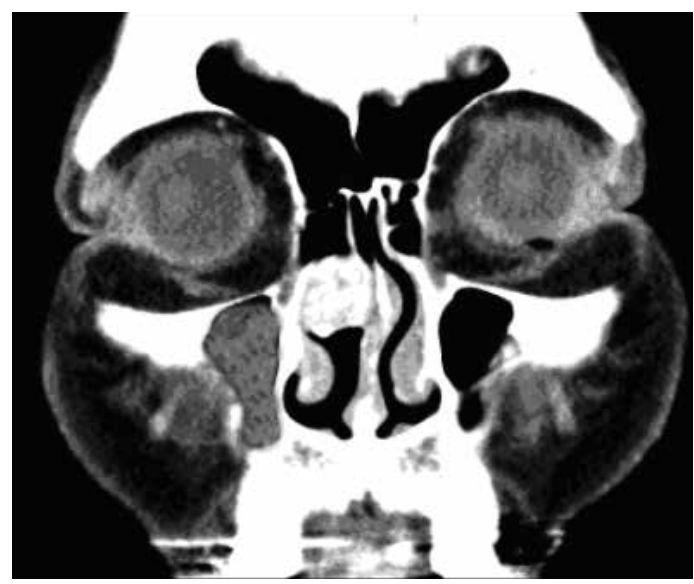

FIG. 2. CT of the sinuses shows one hyperdense lesion of about $1.3 \times 1.2$ $\mathrm{cm}$ over right middle meatus with an opacified right maxillary sinus presented symptoms included epistaxis $(14 / 15,93.3 \%)$, followed by nasal obstruction $(12 / 15,80.0 \%)$, rhinorrhea $(8 / 15$, $53.3 \%)$, protruding tumor $(3 / 15,20.0 \%)$, facial pain $(2 / 15$; $13.3 \%)$, and headache $(1 / 15 ; 6.7 \%)$. The mean duration of symptoms prior to admission was $3.4 \pm 3.9$ months (ranging from 2 weeks to 12 months). The most common site of the lesion was the anterior nasal septum $(6 / 15,40.0 \%)$, followed by the inferior turbinate $(4 / 15,26.6 \%)$, nasal vestibule $(3 / 15$, $20.0 \%)$, posterior nasal septum $(1 / 15,6.7 \%)$ (Figure 1), and middle turbinate $(1 / 15,6.7 \%)$.

Rigid endoscopy found a single dark-red to perse polypoid mass which bled easily when the lesion was touched by a telescope. On CT examinations, the lesions were all characterized by the presence of well-defined, enhancing soft tissue density without bony erosions. Maxillary sinusitis was found in two patients $(13.3 \%)$. Among them, one had a lobular capillary hemangioma over the middle turbinate (Figure 2) and the other had a hemangioma over the inferior turbinate. All these patients underwent surgical removal of the hemangioma. While three patients with a lesion over the nasal vestibule underwent classical local excision, the others underwent endoscopic excisional surgery. There were no postoperative complications observed. The follow-up periods ranged from 6 to 75 months, with the mean of $27.3 \pm 18.7$ months. No recurrence was observed in the present case series.

\section{DISCUSSION}

Lobular capillary hemangioma was first described as human botryomycosis by Poncet and Dor in 1897 (2). In the practice of otolaryngology, lobular capillary hemangioma is a benign vascular lesion commonly occurring in the skin and 
mucosa of the oral cavity, and is less commonly found in the nasal cavity. Proposed contributing factors for the development of lobular capillary hemangioma include trauma, hormones, viral oncogenes, arteriovenous malformations, and angiogenic growth factors (3). In the present series, underlying rheumatoid arthritis in one patient was considered to be the triggering factor of the nasal lobular capillary hemangioma. The increased blood levels of angiogenic growth factors in rheumatoid arthritis patients, namely vascular endothelial growth factor (VEGF) and basic fibroblast growth factor (bFGF), may be critical in triggering the formation of hemangiomas (4).

According to the literature, lobular capillary hemangioma of the nasal cavity show a female predominance, and most commonly occur in the third decade of life (5). In our series, although two-thirds of the patients were female, no peak incidence was found in the third decade. Nearly $75 \%$ of the patients in this study were between 20 and 60 years of age.

Epistaxis was the most common presenting symptom in this series, followed by other intra-nasal symptoms including nasal obstruction and rhinorrhea. Extra-nasal symptoms such as facial pain and headache were less commonly observed. Puxeddu et al. (6) reported that the most common site of a nasal lobular capillary hemangioma is the nasal septum $(55.0 \%)$, followed by the nasal vestibule $(17.5 \%)$, inferior turbinate $(12.5 \%)$, middle turbinate $(7.5 \%)$, and uncinate pro$\operatorname{cess}(7.5 \%)$. This is consistent with our results showing that the nasal septum, especially the anterior region, was the most frequent occurring site.

In view of the similar clinical features of the intranasal mass, the differential diagnosis should include inflammatory lesions such as sarcoid, Wegener's granulomatosis, neoplastic lesions such as sinonasal papillomas, hemangiopericytoma, histiocytoma, leiomyoma, osteoma, squamous cell carcinoma, adenocarcinoma, esthesioneuroblastoma, angiosarcoma, and even foreign bodies (7). CT examination or magnetic resonance imaging (MRI) can be applied to exclude bony erosions or considerations of malignant formations. In this study, all lesions presented as enhancing soft tissue without bony erosions under CT examination. The tumor may also affect the patency of the ostiomeatal complex, leading to poor sinus drainage and maxillary sinusitis.

Surgical excision is the preferred treatment for lobular capillary hemangioma of the nasal cavity. The surgical methods used for this lesion include electrocoagulation, cryotherapy, LASER, excisional surgery, and excisional surgery following angiography with embolization $(6,8)$. In our study, all patients underwent excisional surgery with classical local excision or endoscopic excisional surgery without preoperative embolization. The recurrence rate for hemangioma ranges from $0 \%$ to $42.0 \%$, depending on the case series and the duration of follow-up $(6,8,9)$. On the basis of 6 to 75 months (mean of 27.3 months) of follow-up, no complication or recurrence was found in our series.

The present study found that lobular capillary hemangioma of the nasal cavity is an uncommon disease in the practice of otolaryngology, and the incidence is higher among females than males in Taiwan. When lobular capillary hemangiomas occurred in the nasal cavity, epistaxis and nasal obstruction were the common initial presenting symptoms, and the anterior region of the nasal septum was the most frequent lesion site. According to the CT findings, all patients presented with well-defined, enhancing soft tissue density lesions without bony erosions. All were successfully treated with classical local excision or endoscopic excisional surgery and no complications or recurrence were observed.

\section{Ethics Committee Approval: N/A.}

Informed Consent: Written informed consent was obtained from patients who participated in this study.

Peer-review: Externally peer-reviewed.

Author contributions: Concept - T-H.C.; Design - T-H.C.; Supervision - C-H.Y., S-T.C.; Resource -T-H.C., C-H.Y., S-T.C.; Materials - T-H.C., S-T.C.; Data Collection\&/or Processing - T-H.C.; Analysis\&/or Interpretation -T-H.C., C-H.Y.; Literature Search - T-H.C.; Writing - T-H.C.; Critical Reviews - C-H.Y., S-T.C.

Conflict of Interest: No conflict of interest was declared by the authors.

Financial Disclosure: The authors declared that this study has received no financial support.

\section{REFERENCES}

1. Kurtaran H, Uraldi C, Ark N, Aktas D. Lobular capillary haemangioma of the middle turbinate. Acta Otolaryngol 2006;126:442-44. [CrossRef]

2. Akyol MU, Yalciner EG, Dogan AI. Pyogenic granuloma (lobular capillary hemangioma) of the tongue. Int $J$ Pediatr Otorhinolaryngol 2001;58:239-41. [CrossRef]

3. Genc S, Surkcuoglu S, Karabulut K, Acar B, Tuncel U, Degerli S. Giant lobular capillary hemangioma of the nasal septum. Turk J Med Sci 2009;39:325-8.

4. Nagashima M, Asano G, Yoshino S. Imbalance in production between vascular endothelial growth factor and endostatin in patients with rheumatoid arthritis. $J$ Rheumatol 2000;27:2339-42.

5. Ozcan C, Apa DD, Gorur K. Pediatric lobular capillary hemangioma of the nasal cavity. Eur Arch Otorhinolarygol 2004;261:449-51. [CrossRef]

6. Puxeddu R, Berlucchi M, Ledda GP, Parodo G, Farina D, Nicolai P. Lobular capillary hemangioma of the nasal cavity: A retrospective study on 40 patients. Am J Rhinol 2006;20:480-4. [CrossRef]

7. Miller FR, D'agostino MA, Schlack K. Lobular capillary hemangioma of the nasal cavity. Otolaryngol Head Neck Surg 1999;120:783-4. [CrossRef]

8. El-Sayed Y, Al-Serhani A. Lobular capillary haemangioma (pyogenic granuloma) of the nose. J Laryngol Otol 1997;111:941-5. [CrossRef]

9. Smith SC, Patel RM, Lucas DR, McHugh JB. Sinonasal lobular capillary hemangioma: a clinicopathologic study of 34 cases characterizing potential for local recurrence. Head Neck Pathol 2013;7:129-34. [CrossRef] 